\title{
The ITHEPHY project and its software platform: enhancing remote teacher-student collaboration
}

\author{
Gianluca Peco ${ }^{1, *}$, Angelo Carbone ${ }^{1,2}$, Sébastien Gadrat ${ }^{3}$, and Jean Orloff ${ }^{4}$ \\ ${ }^{1}$ INFN Bologna, Viale Berti Pichat 6/2, 40127 Bologna, Italy. \\ ${ }^{2}$ University of Bologna, Viale Berti Pichat 6/2 40127 Bologna, Italy. \\ ${ }^{3}$ IN2P3 Computing Centre, 21 Avenue Pierre de Coubertin 69100 Villeurbanne, France. \\ ${ }^{4}$ Université Clermont Auvergne, 49 bd Francois Mitterrand CS60032 63001 Clermont-Ferrand, France.
}

\begin{abstract}
.
iTHEPHY is an EU strategic partnership ERASMUS+ project aiming at developing innovative student-centred Deeper Learning Approaches (DPA) for students enrolled in particle physics master's degree programmes. This paper describes the iTHEPHY project status and main goals attained, with a focus on a web-based virtual environment developed to support groups of students and teams of teachers during their DPA learning and teaching activities. The platform is described in detail, focusing on the methodologies and technologies which enabled to deliver a modular, user-friendly, open-source, scalable and reusable platform. Identity and Access Management are the building blocks installed for the iTHEPHY project. Different configurations of services in the cloud will be also covered.
\end{abstract}

\section{Introduction}

iTHEPHY is EU funded project involving universities and research institutes from Italy, Germany and France. The Consortium includes: Alma Mater Studiorum - Università di Bologna (UNIBO) Bologna, Italy; Unversité Clermont Auvergne (UCA), Clermont-Ferrand, France; Technische Univeristät Dortmund (TUD), Dortmund, Germany; Istituto Nazionale di Fisica Nucleare (INFN), Italy; Centre National de La Recherche Scientifique and Institut National de Physique Nucleaire et de Physique de Particules (CNRS/IN2P3), France; Deutsches Elektronen-Synchrotron Laboratory (DESY), Hamburg, Germany.

The project aims at building a strategic partnership to promote an innovative educational environment and exchange of good practices in the field of higher education. The project consists developing e-learning tools for teaching particle physics to students attending the second year of the master degree, making them work together in real research projects supervised by international teachers and researchers belonging to the iTHEPHY Consortium. The innovative educational approach proposed promotes internationalization, team-work skills and boosting mobility worldwide. The project foresees three so-called Intellectual Outputs:

1. a web-based platform to support the team during the project which integrates a video web-conference plug-in, a chat room, a shared storage area for files, a scheduler/agenda

*e-mail: gianluca.peco@bo.infn.it 
for planning meetings between teachers and students, and a project management tool for an efficient implementation of projects, able to assign sub-tasks and tracking their progress;

2. guided exercises with full solutions on an e-learning platform;

3. a handbook describing the implementation of the project for future replications.

\section{Motivation and Genesis}

iTHEPHY is a project funded by the European Commission on higher education methodologies with the aim of addressing some of the most urgent priorities, recently pointed out in the Report on Public Consultation of the EU Modernization Agenda for Higher Education (2016) [1]:

- promoting internationalization, recognition and mobility, supporting changes in line with Bologna principles[2]. This priority is justified by an essential need of students enrolled in master courses. After the master degree, students' careers normally evolve in an international research context: universities, international research institutes and laboratories, and private industries are the most frequent employers. However, the small number of exchanges are limited by crossing cultural and language boundaries, which prevent students and the master courses from having the right level of internationalization during their studies. The Consortium is realized on the basis of strong national synergies between academic and research institutions, to strengthen the link between teaching and research. Its European flavour, founded on a long-term collaboration between all partners in High Energy Physics (HEP) experiments, such as the ones undertaken at the CERN, motivates strongly the master students to be more open to EU and non-EU mobility. All these characteristics are key ingredients for promoting with success the development of an internationalized mind-set, the recognition, by assigning ECTS (European Credit Transfer System) credits, and the mobility both contributing to overcome language and cultural barriers.

- Enhancing the quality and relevance of students' knowledge and skills. The innovative learning and teaching methodologies promoted by iTHEPHY enhance the quality and the relevance of the student's knowledge and skills. Learning and teaching activities are carried out by teams of highly qualified professors and researchers experienced in international research projects. In this context, both students and teachers will improve their collaboration skills. iTHEPHY organised also two intensive teaching and learning activities, the two edition of ISHEP Spring School, where the pedagogical methodologies have been tested and be adopted in future editions. The new methodologies provide students with the right instruments to develop critical thinking, problem-solving attitude and collaborative perspectives.

- Open and innovative practices in a digital era. A fundamental aspect of the project is the development of ICT tools to support the groups of students and the teams of teachers during their learning and teaching activities. In particular a customized Moodle [3] platform, based on open-source software, has been developed to integrate, on a web-based virtual environment, a standard Moodle installation with additional tools useful for sharing documents, meeting virtually trough chat/video rooms, tracking projects and scheduling meetings. 


\section{Educating-Trough-Research: The Tandem-Project}

Educating-trough-research is one of core teaching, and learning activity of the iTHEPHY project. One of this activity is Tandem-Project (TP). Figure 2 shows the logo used to advertise the activities among students. This innovative educational methodology was for the first time tested starting in October 2018. Eighteen students, enrolled in the second year of the master's degree in physics from the three partner universities were engaged to be part of TPs. They were combined to form seven cross-national teams (made of two or three students and two or three supervisors). International research projects based on HEP research were assigned to each group. To achieve the expected results, students and supervisors organized regular virtual meetings (bi-weekly). Also, two video-conferencing meetings were organized, gathering all the students and supervisors together to share the status of their research. The project-based learning and teaching activities were supported by advanced collaborations, integrated into a customized Moodle platform built with open-source software. Figure. 1 shows a view of the Moodle web page, in particular the main page of the Summer School held on May-June 2020.

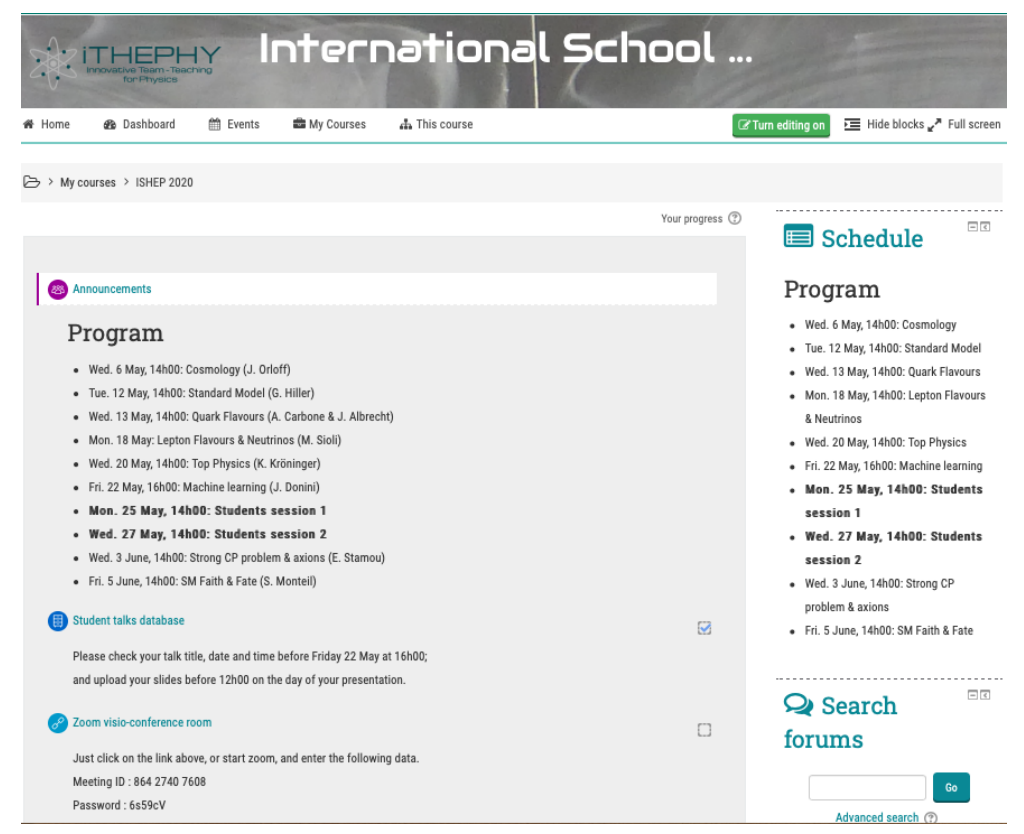

Figure 1. A view of the Moodle web page.

The standard Moodle installation has been extended with the integration of additional educational tools. In more details a TP consist

- defining

a project: a small research project from HEP context, like data analysis, a simulation of a detector, etc.;

a target: a result to be presented at the end of the project;

a time-line: organizing a calendar, scheduling meetings, considering all the intermediate steps to achieve the expected results, indicating when the results are ready to be shown to other colleagues and supervisors; 


\section{- realizing:}

a presentation with final results to be presented at the ISHEP Spring School;

a report, following the scheme of a scientific paper, where students describe in details methodologies and results.

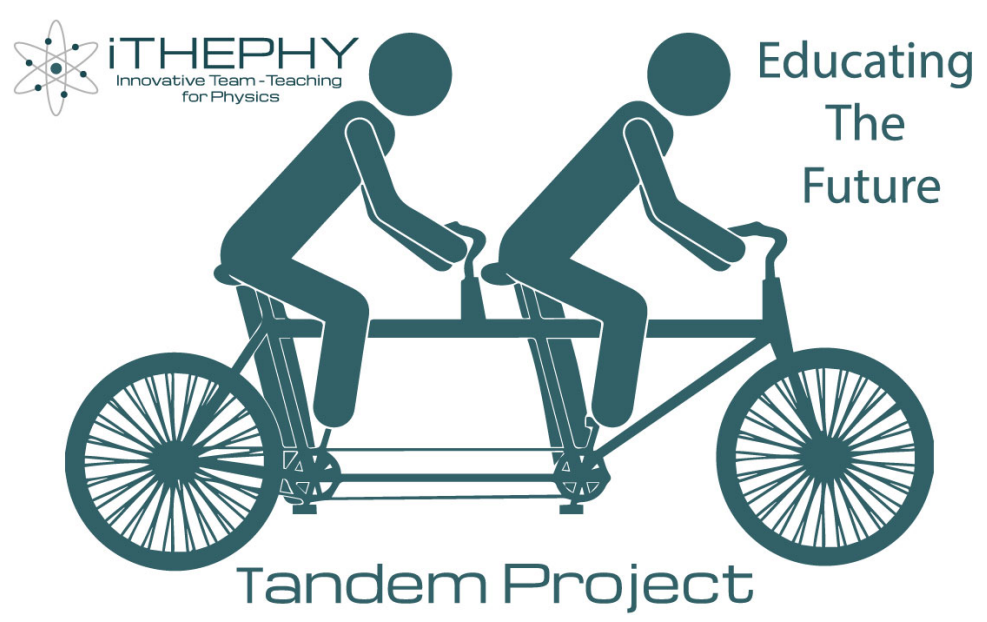

Figure 2. Tandem-Project logo.

A team of international supervisors guided students during the various TPs, exploiting the ICT tools developed in the first part of the project which allowed them to communicate, exchange files, documents and ideas. When all these activities have been completed, and the reports delivered, six ECTS have been awarded by UNIBO to the students, from the three Universities of the Consortium. Supervisors were extremely motivated as most of the projects might continuous as a thesis project. Unfortunately, no official recognition of teaching hours has been assigned by Universities to the supervisors so far. However, there are plans for future acknowledgement. Thanks to extra funds received by the University of Bologna under the project "Bando Strutture 2018", two non-EU students, from the Higher School of Economics (Moscow, Russia) and the Universidad Nacional de Colombia (Bogota, Colombia) participated to the school.

\section{The E-learning Platform}

The e-learning platform is one of the ICT tool developed under the iTHEPHY project based on Infrastructure as a Service (IaaS). It mainly consists a framework where various educational services (open-source) are integrated in a unique virtual space. The platform has been developed in the HEP context, but it can be adopted by all Science Technology Engineering Mathematics disciplines. To develop the proper ICT tools for iTHEPHY, it was necessary to implement and test additional functionalities on top to a standard Moodle installation, namely:

- a video web-conference plugin for meetings, remote lectures, live events;

- a chat room to have a real-time, quick and synchronous discussion; 
- a shared area, as central resource repository to share files, documents and notes related to the project;

- an appointment scheduler for planning meetings between teachers and students and between students;

- a project management tool for implementing projects, assigning sub-tasks and tracking their progress, assigning roles to team members, giving reports about the progress of the project, handling with gantt chart.

Some of these functionalities are not available in the public releases and additional development was needed to be efficiently integrated in a unique web platform. Software integration has been realized using standard programming language, such as HTML5, CSS3, PHP, and PYTHON. The building blocks shown in Fig. 3 and integrated in a unique virtual space consist of:

- Moodle [10] as LMS, used worldwide for educational purposes;

- BigBlueButton [11], a Moodle plug-in, that allows users to create interactive video lessons and webinars, and to record them;

- Rocket.Chat [12], a chat system with channels and rooms integrated with Jitsi (video conference) and BigBlueButton;

- Redmine [13], a project management software with calendar, agenda and gantt charts functionalities;

- Sharelatex [14], a real-time shared latex editing platform.

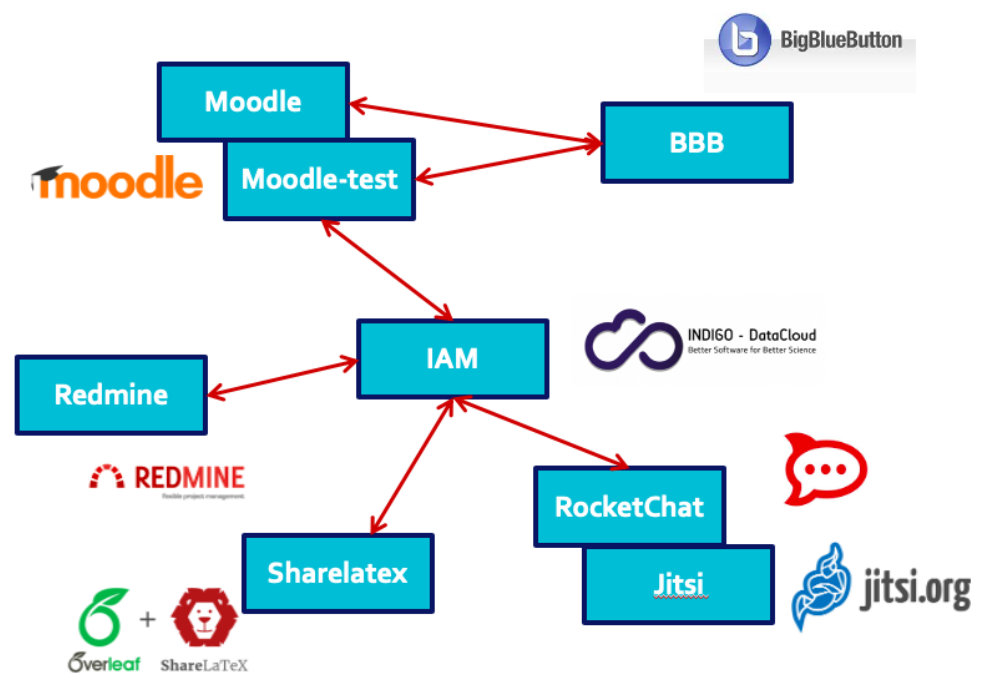

Figure 3. iTHEPHY Platform Building Blocks.

The front-end applications include an authentication system based on Indigo DataCloud [15] IAM using OAuth2 protocol. In contrast, the back-end ones are authenticated trough a JWT API call by the front-end interfaces. Thanks to Indigo DataCloud IAM students and teachers can take advantage of a standard authorization and user management system. The 
platform has been installed in two versions: one is a stable release for the production deployment, it is updated annually (except for security updates which are applied when necessary), while the second version is used for testing and development. The BigBlueButton software has been chosen because it can be easily integrated into Moodle, it has interesting features such as the use of multi-platform HTML5 webrtc clients and the integration of Etherpads for shared notes together with a whiteboard for interactive lectures. Rocket.Chat is a chat system which has all the requirements needed by the project, such as easy integration with Jitsi and BigBlueButton. Jitsi fully respects the platform requirements for audio and video conferencing; it is technologically advanced and widely supported. Sharelatex/Overleaf is the only solution for shared editing in latex so far. INDIGO DataCloud IAM authentication system allowed us to manage authorizations and authentications in an integrated and convenient way. It exploits OAuth2/OpenID-Connect for applications and provides a module for federation integration (IDEM/EDUGAIN). Redmine is a project management system. Even if other competitors exist, the choice was driven by the fact that it is open-source software and provides all the necessary features needed.

\section{Cloud Migration and Software Maintenance}

Cloud services (both private and public) are evolving rapidly and efficiently and the need for users (teachers and students) to have fast, easy and highly flexible deployment systems encouraged the Consortium to build up a microservices based PaaS platform. Natural choices were Kubernetes[16] and Rancher [17]. These tools exploit the HELM Charts [18] for rapid deployment and there is a large number of recipes publicly available and providing integrated monitoring and logging functionalities. Other useful features are Load Balancer ingress, automatic NGINX, integrated Letsencrypt certificates, integrated resource provisioning and RBAC (Role Based Access Control).

Two platforms are deployed: the Test Platform and the Production Platform. The iTHEPHY test platform is installed inside the INFN private cloud infrastructure Cloud@CNAF, where the Openstack resources are instantiated manually and added to the Rancher cluster using a script developed for this purpose. All the resources needed (floating IP, security groups, L4 load balancers) are configured separately and supplied to Rancher from the outside. Permanent volume class provided by Longhorn are used as persistent storage. Longhorn is a $\mathrm{k} 8 \mathrm{~s}$ application that manages cluster nodes and local disks to provide replication. The system is a beta testing implementation running since months without showing any significant issue. The applications installed so far are Moodle, RedMine, Rocket.Chat, Letsencrypt and Longhorn. Soon the IAM authentication system (INDIGO-DataCloud), Jitsi and BigBlueButton will be also added.

The production platform has been deployed at the INFN Corporate Cloud. It is Hybrid cloud environment where all resources are automatically provisioned in a dynamic way. Applications were deployed using Rancher and HELM catalogues. In this configuration, public applications are reachable from the general Internet via Openstack L4 and/or Rancher L7 load balancer and proxies by Rancher nginx service. All necessary security group configurations are made from application receipts. Moodle, Redmine, Rocket.Chat from official HELM repository are installed and configured. In the near future, it will be necessary to create, test and distribute a chart or a YAML for the configuration of environment starting from docker-compose receipts of Jitsi, BigBlueButton and the IAM authentication system. 


\section{Conclusions}

The iTHEPHY project proposes an innovative and unconventional approach of learning and teaching based on educating-through-research activities. This approach is complementary to face-to-face lectures and promotes internationalization of master degrees in and outside Europe. Constant interaction between students and tutors is guaranteed through the exploitation of a customized e-learning platform based on well established technologies such as Moodle [3], Jitsi [5], BigBlueButton [6], INDIGO-DataCloud [15], Redmine [4] and Rocket.Chat [7]. The platform was developed with the aim of supporting the educational objectives: all the software products adopted are FOSS (Free and Open Source Software). Their choice was driven by the solidity of the developer community behind the various projects . Moreover, to facilitate the installation, a container system based on docker has been implemented. In the near future, Rancher solutions to build an iTHEPHY service platform will be released, facilitating the access to the e-learning platform to a wide audience. During the 2020 edition of the ISHEP Spring School, held in Cargese (4-8 May 2020), the ICT tools developed have been successfully adopted, and after the School, they have been deployed.. In addition, the Master Degree in Physics of the iTHEPHY Universities, have included in their educational program the TP activities by granting 6 ECTS.

\section{References}

[1] EU Committee Results of the public consultation on the EU's modernisation agenda for higher education (2016) https://eur-lex.europa.eu/resource.html?uri=cellar: cd0fa1ca-2ee9-11e6-b497-01aa75ed71a1.0001.02/DOC_2\&format=PDF

[2] https://ec.europa.eu/education/policies/higher-education/

bologna-process-and-european-higher-education-area_en

[3] Moodle, https://moodle.org/

[4] Redmine, https://redmine.org

[5] Jitsi, https://jitsi.org

[6] BigBlueButton, https://bigbluebutton.org

[7] Rocket.Chat, https://rocket.chat

[8] Sharelatex, https://www.sharelatex.com

[9] A. Ceccanti, M. Hardt, B. Wegh, A. Millar, M. Caberletti, E. Vianello and S. Licehammer, J. Phys. Conf. Ser. 898 (2017) no.10, 102016. doi:10.1088/1742-6596/898/10/102016

[10] https://moodle.ithephy.eu

[11] https://bbb.ithephy.eu

[12] https://rocket.ithephy.eu

[13] https://redmine.ithephy.eu

[14] http://sharelatex-test-ithephy.in2p3.fr

[15] https://www.indigo-datacloud.eu/

[16] https://kubernetes.io/

[17] https://rancher.com/

[18] https://helm.sh/ 